\title{
ISR Experiment at A1-Collaboration
}

\author{
Miha Mihovilovič ${ }^{1,2, \star}$ and Harald Merkel ${ }^{1}$ for A1-Collaboration \\ ${ }^{1}$ Institut für Kernphysik, Johannes Gutenberg-Universität Mainz, DE-55128 Mainz, Germany \\ ${ }^{2}$ Jožef Stefan Institute, SI-1000 Ljubljana, Slovenia
}

\begin{abstract}
The discrepancy between the proton charge radius extracted from the muonic hydrogen Lamb shift measurement and the best present value obtained from the elastic scattering experiments, remains unexplained and represents a burning problem of today's nuclear physics. In a pursuit of reconciling the puzzle an experiment is underway at MAMI, which exploits the radiative tail of the elastic peak to study the properties of electromagnetic processes and to extract the proton charge form factor $\left(G_{E}^{p}\right)$ at extremely small $Q^{2}$. This paper reports on the latest results of the first such measurement performed at the three-spectrometer facility of the A1-Collaboration, which led to a precise validation of radiative corrections far away from elastic line and provided measurements of $G_{E}^{p}$ for $0.001 \leq Q^{2} \leq 0.017(\mathrm{GeV} / c)^{2}$.
\end{abstract}

\section{Introduction}

The proton has been studied since the early days of experimental hadronic physics [1]. Through the years many different measurements of its properties have been performed, ranging from the pioneering experiments in the 1960s to the high precision measurements done in the last years. In particular, its radius has been determined by various electron scattering experiments and many atomic Lamb shift measurements (see Figure 1). Both approaches gave consistent results. Unfortunately their average does not agree with the findings of the very precise Lamb shift measurements in muonichydrogen $[2,3]$, which report a $6 \sigma$ smaller value for the proton charge radius. The observed discrepancy created a great excitement in the physics community, because it puts QED and our understanding of the nuclear physics to a rigorous test. Since the observation of the inconsistency in 2010, various explanations for it have been offered. The most trivial explanation would be an unidentified error in the existing measurements. However, both spectroscopic and nuclear scattering measurements have been reexamined and no problem has yet been found. Furthermore, all measurements except the muonic-hydrogen measurement were done by different groups and they all mutually agree. However, if it turns out, that the old measurements indeed are erroneous, then a new value of the radius would command also a different value of the Rydberg constant (due to a strong correlation between the two parameters). This would have a strong impact on all existing spectroscopic results [4]. A mistake in the existing calculations used to interpret spectroscopic measurements or an overlooked higher order term also do not appear to be a plausible scenario. The calculations were made by several theoretical groups using different approaches and they all get very similar results [5]. They invested a large effort

\footnotetext{
^e-mail: miha.mihovilovic@ijs.si
} 


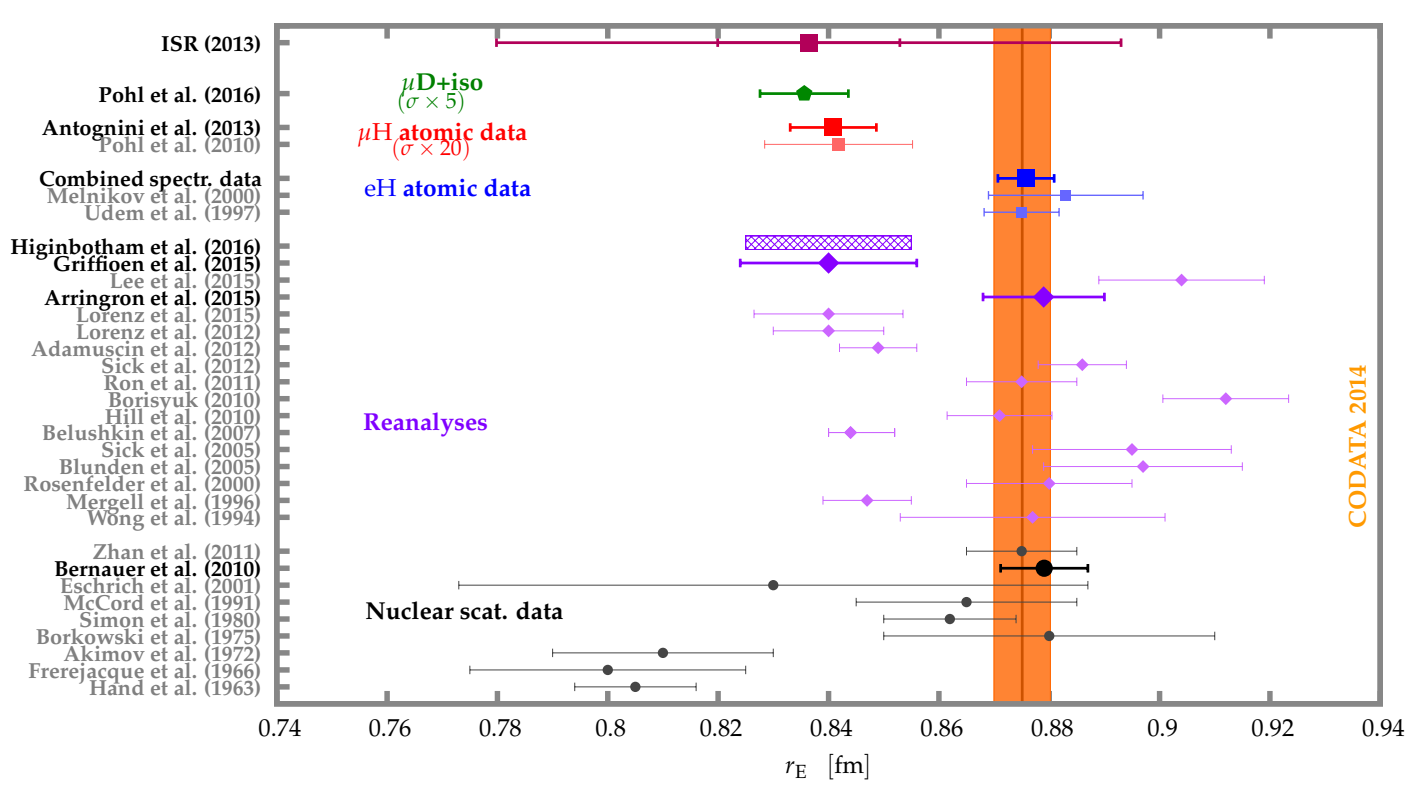

Figure 1. An overview of the available proton charge radius measurements. Full circles show findings of the scattering experiments. Full squares represent values obtained from the Lamb shift spectroscopy. The values determined from the muonic hydrogen (deuterium) measurements are colored red (green). The uncertainties of muonic hydrogen (deuterium) data are multiplied by factor 20 (5) for clarity [6]. The value extracted from the ISR experiment is shown with the purple square at the top of the plot [7].

to examine many different Feynman diagrams and the current consensus is that a missing higher order term is not responsible for the discrepancy, because they all contribute much less than the needed $300 \mu \mathrm{V}$ [8]. Hence, this could suggest a hidden problem with a leading (vacuum polarization) term, or it could even be an indication of a fundamental problem with QED. People have offered also many other explanations, ranging from the incomplete two-photon corrections to the contributions of the molecular ions. However, the most intriguing ideas are those claiming that the discrepancy can not be explained within the framework of Standard Model. The idea of a new force and corresponding mediator particle that breaks the electron-muon universality is interesting also because it could simultaneously explain the muonic $(g-2)_{\mu}$ puzzle [9]. A promising candidate for a mediator particle is a $\mathrm{U}(1)$ gauge boson that moderates the interaction between the dark matter and the standard model particles. Unfortunately, no such particle has been found yet [10].

In order to overcome the present impasse, further measurements are needed. In this paper we describe a new kind of nuclear scattering experiment which has a potential to provide precise formfactor data at $Q^{2}<0.004 \mathrm{GeV}^{2} / c^{2}$ and thus ensure a more robust extraction of the proton charge radius from the nuclear experiments.

\section{Radius via cross-section measurement}

In a typical scattering experiment is the radius of a proton determined indirectly by measuring the cross-section for elastic scattering of electrons on hydrogen. The measured cross-section depends on electric and magnetic form-factors, $G_{E}^{p}$ and $G_{M}^{p}$, which carry information about the charge and 
magnetization distribution in the proton and are extracted from the measured data via Rosenbluth separation. The charge radius is extracted from the slope of the electric form-factor at $Q^{2}=0$ :

$$
r_{E}^{2} \equiv-\left.6 \hbar^{2} \frac{d}{d Q^{2}} G_{E}\left(Q^{2}\right)\right|_{Q^{2}=0},
$$

where $Q^{2}$ represents the square of the momentum transfer four-vector. The world collection of data on the $G_{E}^{p}$ that we have available at low $Q^{2}$ are shown in Fig. 2. There you can see, that the existing measurements are already very precise, but the problem is, that even the best experiments provide data only down to $Q^{2}=0.004(\mathrm{GeV} / c)^{2}$. Consequently, the data need to be extrapolated towards $Q^{2}=0$ in order to estimate the radius. Since such extrapolations tend to be unstable they can lead to a systematic offset of the extracted value for the proton charge radius. The only way to overcome this problem is to provide new measurements at even smaller $Q^{2}$, investigate the real behaviour of the $G_{E}^{p}$ at $Q^{2} \rightarrow 0$ and ensure more accurate absolute normalisation of the data. However, the measurements at $Q^{2}<0.004 \mathrm{GeV}^{2} / c^{2}$ are extremely difficult due to the kinematical limitations of the available apparatuses. To reach this kinematical region, the presented experiment uses not only the elastic data, but exploits also the information about the $G_{E}^{p}$ that is hidden inside the radiative tail of the elastic peak.

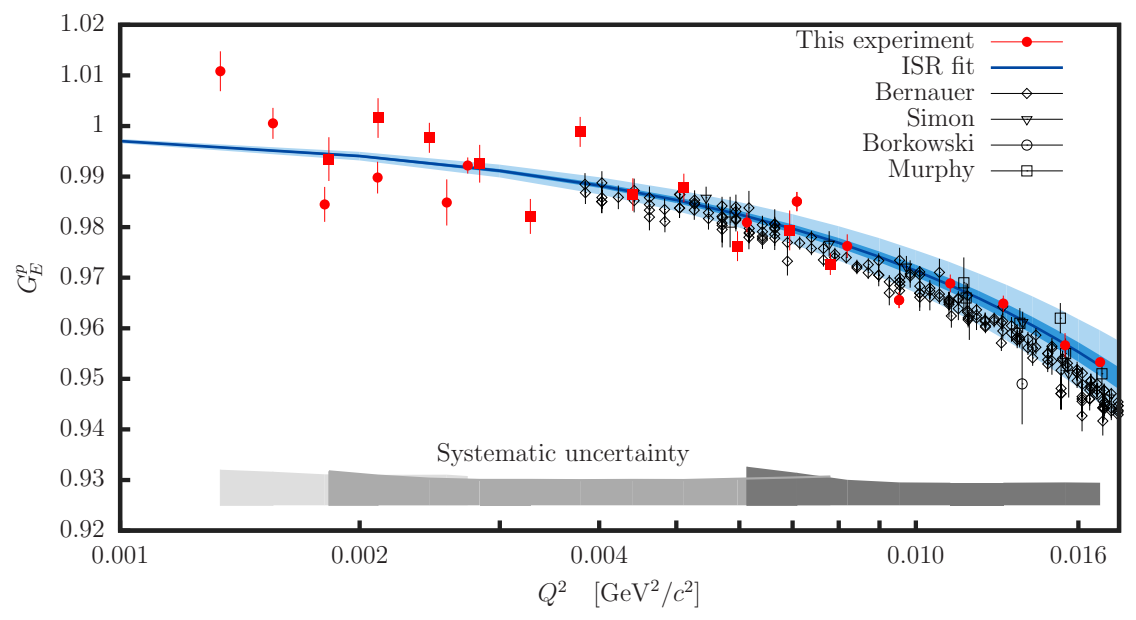

Figure 2. The proton electric form factor as a function of $Q^{2}\left(=Q_{\text {Out }}^{2}\right)$. Empty black points show previous data. The results of this experiment are shown with full red circles. The error bars show statistical uncertainties. Grey structures at the bottom shows the systematic uncertainties for the three energy settings. The curve corresponds to a polynomial fit to the data defined by Eq. (1). The inner and the outer bands around the fit show its uncertainties, caused by the statistical and systematic uncertainties of the data, respectively. See Ref. [7] and references within.

\section{Initial-state radiation technique}

The radiative tail of an elastic peak observed in the measured nuclear spectra is dominated by contributions of two Bethe-Heitler diagrams [11] shown in Figure 3. The initial state radiation diagram (BH i) describes the process where the incident electron emits a real photon before interacting with the proton. Since the emitted photon carries away part of the incident energy, the momentum transferred to the proton $\left(Q_{\mathrm{Vertex}}^{2}\right)$ is decreased. Hence, this process opens the possibility to probe the proton 
structure at values of $Q_{\text {Det }}^{2}=Q_{\text {Vertex }}^{2}$ that are smaller than the value fixed by the experimental kinematics. On the other hand, the final state radiation diagram (BH f) corresponds to the reaction where the real photon is emitted only after the interaction with the nucleon. Consequently, $Q_{\mathrm{Vertex}}^{2}$ at the vertex remains constant, while the detected $Q_{\text {Det }}^{2} \leq Q_{\text {Vertex }}^{2}$ changes.

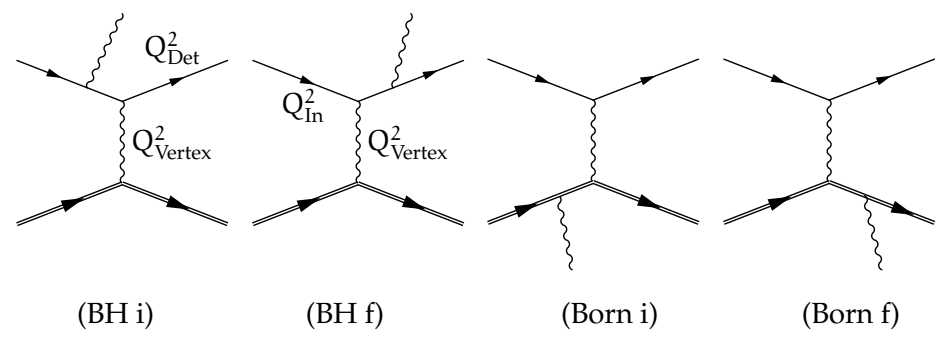

Figure 3. Feynman diagrams for inelastic scattering of electron off a proton, where the electron and proton emit real photons before or after the interaction. Diagrams where electrons emit a photon are known as Bethe-Heitler (BH) diagrams, while those where protons emit real photons are called Born diagrams. The $Q_{\text {Vertex }}^{2}$ represents the square of the four-momentum transferred to the hadron. The $Q_{\mathrm{In}}^{2}$ is the momentum fixed by the beam energy and the scattering angle, while the $Q_{\mathrm{Det}}^{2} \leq Q_{\mathrm{In}}^{2}$ corresponds to value measured with the detector. For the (BH-i) $Q_{\text {Vertex }}^{2}=Q_{\text {Det }}^{2}$, and for the (BH-f) $Q_{\text {Vertex }}^{2}=Q_{\mathrm{In}}^{2}$.

In an experiment only $Q_{\text {Det }}^{2}$ can be measured, which means that looking only at data, initial state radiation processes can not be distinguished from the final state radiation. To reach information corresponding only to the initial state radiation, measurements need to be studied together with results of a Monte-Carlo simulation. This is the basic idea of the discussed MAMI experiment, which aims to extract $G_{E}^{p}$ in the range $10^{-4} \leq Q_{\text {Vertex }}^{2} \leq 0.005(\mathrm{GeV} / c)^{2}$.

\section{Experiment}

First full measurement of this type was performed in 2013 at the spectrometer hall of the A1Collaboration. For the measurement rastered electron beam with energies of $195 \mathrm{MeV}, 330 \mathrm{MeV}$ and $495 \mathrm{MeV}$ was used in combination with a liquid Hydrogen target. For cross-section measurements spectrometer B was employed. It was positioned at a fixed angle of $15.25^{\circ}$, while its momentum settings were being adjusted to scan the complete radiative tail for each energy setting. Kinematic settings were chosen such that the radiative tails of all three setting overlapped. In total, 60 different setups were investigated, resulting in three weeks of data taking: two weeks for production measurements and one week for empty-cell measurements, needed for proper background subtraction. Beam currents were between $10 \mathrm{nA}$ and $1 \mu \mathrm{A}$, depending on the setup and were measured by two probes: the non-invasive Förster probe and the invasive pA-meter. Unfortunately at low beam currents and low beam energies neither of the probes was accurate enough. Hence, spectrometer A, positioned at a fixed setting, was employed for precise monitoring of beam luminosity.

The experiment successfully recorded data for $Q^{2}$ ranging from $2 \cdot 10^{-2}(\mathrm{GeV} / c)^{2}$ down to only 2 . $10^{-4}(\mathrm{GeV} / c)^{2}$ without major experimental difficulties. Unfortunately only a detailed offline analysis then revealed hidden backgrounds coming from the bulk material of the target and the entrance flange of the spectrometer. Elastic events which are a priori outside the spectrometer's acceptance and were blocked by the bulk material of the spectrometer and target, underwent a secondary scattering process in the metal and rescattered back into its acceptance. While the effect was tiny at high energies, these 


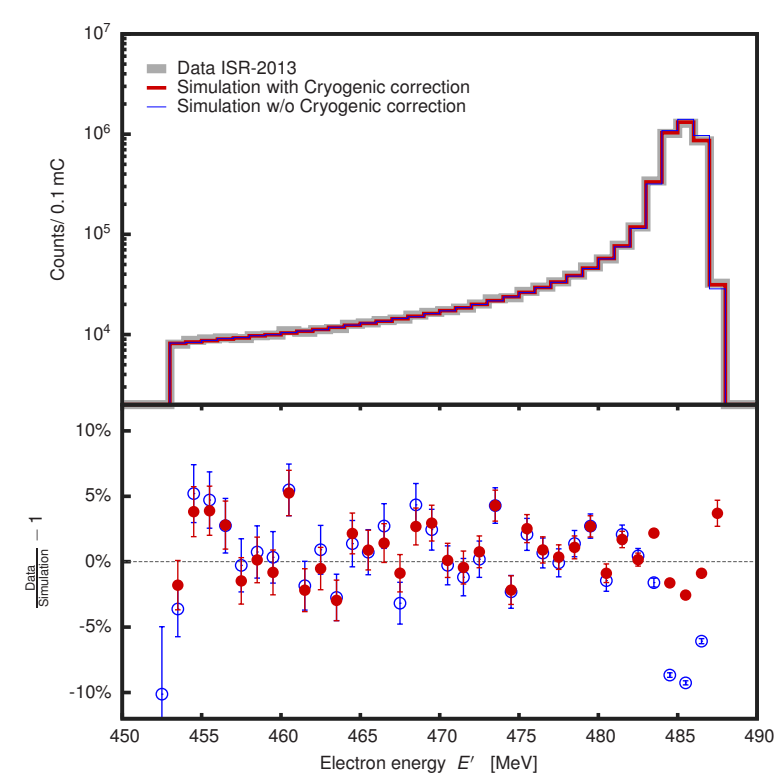

Figure 4. Top: Comparison of the data (grey line) to the simulation for the elastic setting at $495 \mathrm{MeV}$, normalised to the accumulated charge of $0.1 \mathrm{mC}$. The simulation considers $G_{E}^{p}$ given by parameterization of Bernauer [12]. Blue line shows simulation without considering extra cryogenic depositions on the sides. The red line shows the full simulation, which considers much thicker layer of cryogens (mostly frozen nitrogen) on the target's side walls. Bottom: Relative difference between the data and simulation. The empty blue and full red circles show the comparison of the data with the uncorrected and corrected simulation, respectively. Corrected simulation offers much better agreement with the data in the vicinity of the elastic peak.

additional events significantly distorted measured spectra at low energies and consequently limited the analysis to $1 \cdot 10^{-3}(\mathrm{GeV} / c)^{2}$.

\section{Results}

First results of the experiment are presented in Ref. [7]. Besides the backgrounds from the bulk material of the apparatus, which limited its kinematic reach, the experiment also suffered from the cryogenic depositions gathered on the walls of the liquid hydrogen target. They affected the detected rates as well as the energy losses of the particles. While the background contributions to the spectra could be monitored, modelled and corrected for, the energy losses of the detected particles could not be precisely determined. This most dramatically affected the elastic events where the rates changed quickly with the energy of the particle and where any inconsistency could be easily observed. Since in the primary analysis these effects were not under control and the inconsistency between the data and simulation at the top of the elastic peak could not be resolved, the elastic points were omitted from the analysis. Only after the careful inspection of external radiative corrections and effects of collisional energy losses, the discrepancy could be attributed to the different amount of the cryogens on front (back) and side walls of the target cell. Since the material on the side walls is invisible to the spectrometers, the amount of snow had to be estimated by matching the functional dependence of the simulation to the measured elastic spectra. See Fig. 4. It was determined, that the layer of cryogens on the side can be $200 \times$ thicker than at the front (back) of the cell, or roughly $0.1 \mu \mathrm{m}$. This new insight significantly improved the agreement between the data and simulation and allowed us to add elastic data to the analysis.

The complete data set is now shown in Fig. 2. Results exhibit a better than $1 \%$ agreement between the data and simulation in a region of the tail, that extends even more than $200 \mathrm{MeV}$ from the elastic line. This demonstrates that the radiative corrections are understood to a sub percent level, which is essential for all future precision measurements. Furthermore, form-factors extracted from the measured tail are in a good agreement with the values from the previous experiments, which proves, that ISR approach is a valid technique for measuring form-factors at low $Q^{2}$. Experiment also provided 
form-factors in the region $1 \cdot 10^{-3}(\mathrm{GeV} / c)^{2} \leq Q^{2} \leq 4 \cdot 10^{-3}(\mathrm{GeV} / c)^{2}$ where no measurements existed before. The extracted $G_{E}^{p}$ values could again compared to the polynomial [7]

$$
G\left(Q^{2}\right)=1-\frac{r_{p}^{2} Q^{2}}{6 \hbar^{2}}+\frac{a Q^{4}}{120 \hbar^{4}}-\frac{b Q^{6}}{5040 \hbar^{6}},
$$

where parameters $a=(2.59 \pm 0.194) \mathrm{fm}^{4}$ and $b=(29.8 \pm 14.71) \mathrm{fm}^{6}$, which determine the curvature of the fit, were taken from Ref. [13]. The three data sets were fit with a common parameter for the radius, $r_{p}$, but with different renormalisation factors, $n_{E_{0}}$, for each energy. In terms of this fit with 21 degrees of freedom and $\chi^{2}$ of 95.4, the normalisations and the radius were determined to be $n_{195}=$ $1.002 \pm 0.002_{\text {stat }} \pm 0.007_{\text {syst }}, n_{330}=1.000 \pm 0.001_{\text {stat }} \pm 0.003_{\text {syst }}, n_{495}=0.999 \pm 0.001_{\text {stat }} \pm 0.004_{\text {syst }}$, and $r_{p}=\left(0.836 \pm 0.017_{\text {stat }} \pm 0.059_{\text {syst }} \pm 0.003_{\triangle a, \Delta b}\right) \mathrm{fm}$. Due to the presence of the three elastic points, the extracted radius is now a bit larger, but the present systematic uncertainty, which is dominated by the backgrounds from the spectrometer's snout, is still too large to make a significant contribution to the proton radius puzzle, see Fig. 1. However, knowing that the method works is a strong motivation for finding a way to remove the limiting background and for executing a next generation experiment [14].

\section{Conclusions}

The problem of the proton charge radius is a persisting open question of today's physics. Since the available theories are unable to explain the discrepancies between different types of measurements, the present situation could be improved only by doing new measurements in the kinematic regions that are most sensitive to the charge radius. One of the most promising ongoing experiments is the Initial-state radiation experiment at MAMI, which uses new experimental technique based on ISR to extract $G_{E}^{p}$ at very low $Q^{2}$, where no measurements have existed before. Such data could ensure better determination of the absolute normalisation of the nuclear scattering data and further constrain the fits used to extract the radius. The first measurement of this type, presented here, demonstrated, that ISR approach works and has already provided data at $Q^{2} \geq 10^{-3} \mathrm{GeV}^{2} / c^{2}$. To reach the form-factors at $Q^{2} \approx 10^{-4} \mathrm{GeV}^{2} / c^{2}$ the setup needs to be upgraded in order to avoid limiting backgrounds. This will be accomplished in the upcoming experiment with the new hypersonic jet target.

\section{References}

[1] R. W. McAllister and R. Hofstadter, Phys. Rev. 102 , 851 (1956).

[2] R. Pohl et al., Nature 466, 213 (2010).

[3] A. Antognini et al., Science 339, 417 (2013).

[4] R. Pohl et al., Science 353, 6300, 669 (2016).

[5] A. Antognini et al., Annals of Physics 331, 127 (2013).

[6] J. Beringer et al. (Particle Data Group), Phys. Rev. D 86, 010001 (2012).

[7] M. Mihovilovič et al., Phys. Lett. B 771, 194 (2017).

[8] F. Hagelstein et al., Prog. Part. Nucl. Phys. 8829 (2016).

[9] J. C. Martens, J. P. Ralston, arXiv:1606.06209 [hep-ph] (2016).

[10] H. Merkel et al., Phys. Rev. Lett. 112, 221802 (2014).

[11] M. Vanderhaeghen et al., Phys. Rev. C 62, 025501 (2000).

[12] J. C. Bernauer et al., Phys. Rev. C 90, 015206 (2014).

[13] M. O. Distler, J. C. Bernauer and T. Walcher, Phys. Lett. B 696, 343 (2011).

[14] H. Merkel (spokesperson), MAMI proposal A1-02-16, (2016). 